\title{
Endoscopic Transmural Necrosectomy: Timing, Indications, and Methods
}

\author{
Rungsun Rerknimitr ${ }^{1,2}$ \\ ${ }^{1}$ Division of Gastroenterology, Department of Medicine, Faculty of Medicine, Chulalongkorn University and King Chulalongkorn Memorial \\ Hospital, Bangkok, ${ }^{2}$ Center of Excellence for Innovation and Endoscopy in Gastrointestinal Oncology, Chulalongkorn University, Bangkok, \\ Thailand
}

Walled-off necrosis is considered one of the most severe complications after an episode of severe acute pancreatitis. Traditionally, percutaneous drainage is selected as the first treatment step, while open surgery can be planned as a secondary option if necrosectomy is required. In recent years, endoscopic necrosectomy has evolved as a more favorable approach. To date, a step-up treatment strategy is recommended, particularly when a plastic stent is selected as the drainage device. Multi-gateway endoscopic therapy may be used in a step-up fashion if only one stent fails to clear debris. Over many years, there has been an evolution in stent selection, from plastic to metallic stents. Within a few years of its clinical usage, lumen-apposing stents are gaining more popularity as they offer direct endoscopic necrosectomy and only require a few sessions. Clin Endosc 2020;53:49-53

Key Words: Endoscopic necrosectomy; Lumen-apposing metallic stent; Walled-off necrosis

\section{INTRODUCTION}

Walled-off necrosis (WON) is considered one of the most severe complications after an episode of severe acute pancreatitis. Although many patients who suffer from WON can be treated conservatively, a quarter of them may require interventional drainage or debridement. ${ }^{1}$ Traditionally, percutaneous drainage is selected as the first treatment step, while open surgery can be planned as a secondary option, if necrosectomy is required. Due to the aggressiveness of open surgery and risk of pancreatico-cutaneous fistula from the percutaneous approach (unless internal drainage or pancreatic duct bridging by an internal stent is provided), ${ }^{2}$ these two techniques are

Received: July 2, 2019 Revised: August 1, 2019

Accepted: August 22, 2019

Correspondence: Rungsun Rerknimitr

Division of Gastroenterology, Department of Medicine, Faculty of Medicine, Chulalongkorn University and King Chulalongkorn Memorial Hospital, 1873 Rama IV Rd. Lumpini, Bangkok 10330, Thailand

Tel: +66-2-256-4265, Fax: +66-2-252-7839, E-mail: ERCP@live.com

ORCID: https://orcid.org/0000-0001-6866-6886

(c) This is an Open Access article distributed under the terms of the Creative Commons Attribution Non-Commercial License (http://creativecommons.org/ licenses/by-nc/3.0) which permits unrestricted non-commercial use, distribution, and reproduction in any medium, provided the original work is properly cited. not recommended as the first line in current practice. ${ }^{3}$ Over the last decade, new minimally invasive techniques, including video-assisted retroperitoneal debridement (VARD) and endoscopic necrosectomy (EN), have emerged as more favorable approaches. In this review, we will mainly address EN and the selection of patients with WON for this procedure based on the appropriate timing and indications. Additionally, we will elaborate on the different EN techniques and the potential use of new devices, particularly, lumen-apposing metallic stents (LAMSs).

\section{TIMING, INDICATIONS, STRATEGY FOR INTERVENTION, AND ITS LIMITATIONS}

The pathophysiology of acute pancreatitis can be divided into acute interstitial or acute necrotizing pancreatitis. According to the revised Atlanta classification, ${ }^{4}$ at the age of less than 4 weeks, acute peripancreatic collection develops from acute interstitial pancreatitis whereas acute necrotic collection develops from acute necrotizing pancreatitis. While pseudocysts evolve from acute necrotic collection, WON is defined as the 4-week evolution of acute necrotic collection. 
Currently, there are two strategies for WON intervention: direct EN, by performing debridement in the first few sessions regardless of the clinical course of WON; and a step-up approach, in which EN is performed after a failed response to transmural drainage with only stent insertion. More importantly, draining WON after 4-6 weeks, with expected wall maturity after an episode of acute pancreatitis, has been well practiced. Although this timing is arbitrarily recommended, the practice has been adopted based on surgical experience for over 35 years. ${ }^{5}$

Many series ${ }^{6-8}$ have demonstrated the advantage of a stepup strategy to avoid unnecessary EN by showing that about $20 \%-90 \%$ of patients with WON can be treated by endoscopic drainage alone, with either a plastic stent (PS) or fully covered metallic stent, including a LAMS. Many endoscopists may elect to utilize a step-up strategy because they would like the transmural tract to mature a few days after the transmural stent is deployed. Certain factors that might preclude the success of the drainage-only strategy include the large size of WON, the small diameter of the stent, and the higher proportion of solid debris in the cavity. ${ }^{6-8}$ Thus, a patient with large WON or WON with a high proportion of solid debris may be a good candidate for direct EN, and the preferred stent may be a larger-diameter stent such as a LAMS (Fig. 1).

Although endoscopic guidance by examining the bulging area in the stomach or duodenum with a standard endoscope

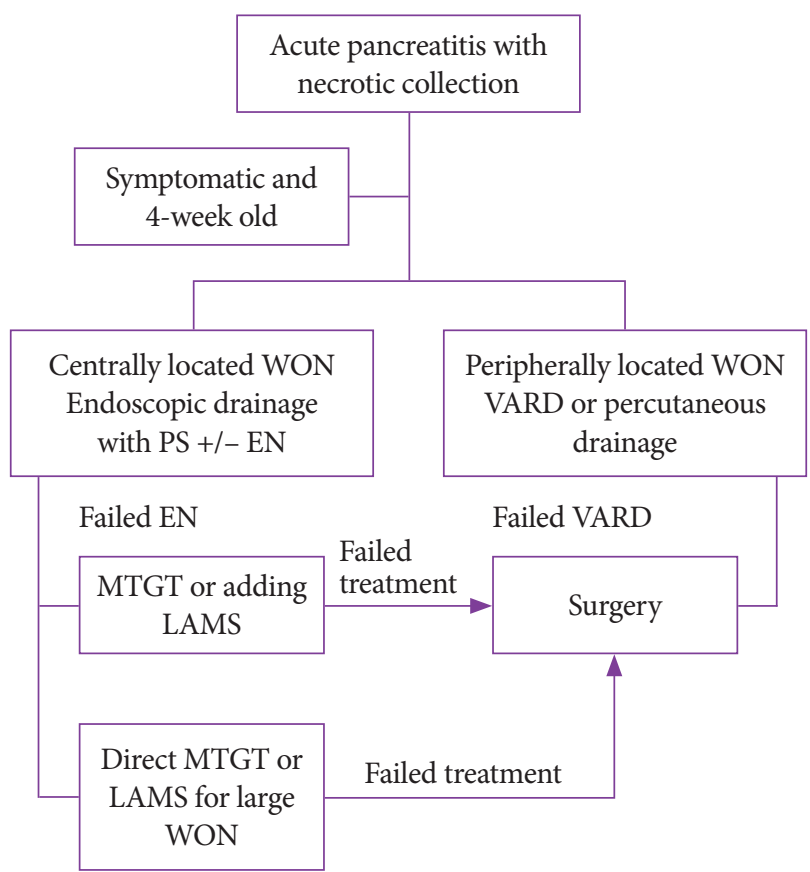

Fig. 1. Algorithm to approach for walled-off necrosis (WON). EN, endoscopic necrosectomy; LAMS, lumen-apposing metallic stent; MTGT, multiple transluminal gateway technique; PS, plastic stent; VARD, video-assisted retroperitoneal debridement. is possible, endosonographic guidance by an echoendoscope has been widely practiced because it can help to avoid interposed blood vessels. Moreover, echoendoscope guidance is capable of targeting non-bulging WON in patients who failed endoscopic visualization. ${ }^{9}$

However, echoendoscope guidance may not be able to access the area beyond the vicinity of an echoendoscope, such as paracolic gutters or WON located at the peripheral sections of the abdomen. Therefore, VARD may be preferred, particularly when WON is located near the left side of the colon and/or under the spleen. ${ }^{10,11}$

Although a landmark trial from the Netherlands demonstrated that EN can reduce the proinflammatory response as well as the composite clinical endpoint when compared with surgical necrosectomy, there were some patients requiring VARD as salvage treatment after failed treatment by EN. Interestingly, their WON was located at the periphery of the abdomen and was approached from the left side. ${ }^{11}$

\section{MULTI-GATEWAY ENDOSCOPIC NECROSECTOMY AND THE ROLE OF LUMEN-APPOSING METALLIC STENT}

The concept of the multiple transluminal gateway technique (MTGT) was first described by Varadarajulu et al. in 2011 to facilitate better drainage of necrotic material and infected fluid from WON. ${ }^{12}$ They recommended the use of echoendoscope-guided puncture for at least two to three tracts, with one tract serving as an irrigation site via a nasocystic catheter. The others work as conduits to drain the contents by having double pigtail stents placed to keep the stomas open. With a back and forth mechanism of fluid drainage in the WON cavity, necrotic material and fluid can be rapidly drained. They reported on 60 patients with WON, 12 patients who underwent the MTGT and 48 patients who had a single PS placed (conventional group). They found that the MTGT provided successful drainage without additional EN in 11/12 patients (91.7\%), whereas the conventional group only achieved successful drainage in 25/48 patients (52\%). Of those 23 patients in the control group who were not successful managed with conventional treatment, 17 patients underwent debridement surgery, 3 patients underwent step-up EN, and 3 patients died because of multiple-organ failure. ${ }^{12}$

In recent years, LAMSs have become the more preferred stent in patients with WON. The advantages of a LAMS over a PS include the larger stent diameter that facilitates the spontaneous dislodgement of large debris and allows end-viewing scope entry for EN. ${ }^{13-15}$ In addition, after LAMS placement, an immediate EN can be performed without waiting for tract 
maturity. ${ }^{15}$ Although, the use of a LAMS is promising, a recent meta-analysis showed no difference in the pooled rates of clinical success and adverse events between WON drained by a LAMS and that drained by a PS $(88.5 \%$ [ $95 \%$ confidence interval, 82.5-92.6; $\left.\mathrm{I}^{2}=71.7\right]$ vs. $88.1 \%$ [95\% confidence interval, 80.5-93.0; $\left.\mathrm{I}^{2}=78.1\right], p=0.93$; and $11.2 \%\left[6.8-17.9 ; \mathrm{I}^{2}=82.0\right]$ vs. $15.9 \%\left[8.4-27.8 ; \mathrm{I}^{2}=78.8\right], p=0.38$, respectively). ${ }^{14}$ Notably, there was significant heterogeneity in the recruited population, and the severity of adverse events developed from a LAMS versus a PS could not be classified and compared directly.

We have recently adopted two concepts and combined them together (MTGT with multiple LAMSs) as we believe that they work through different mechanisms. We had put two LAMSs in a few patients with large WON $(>10 \mathrm{~cm})$ and experienced a very rapid clearance of necrotic tissue within only a few sessions of direct EN (Fig. 2).

\section{THE ENDPOINT FOR ENDOSCOPIC NECROSECTOMY}

The requirement for transmural stents ends after certain objectives have been accomplished, such as the absence or control of ongoing infections, at which point antibiotics can be discontinued. The last step of EN is to remove the stent and close-down the stoma. However, this can be done after confirmation via a pancreatogram to demonstrate no pancreatic duct disruption. The risk of recurrent fluid collection is significant in patients who have pancreatic duct disruption, particularly in those with disconnected duct syndrome. ${ }^{16} \mathrm{~A}$
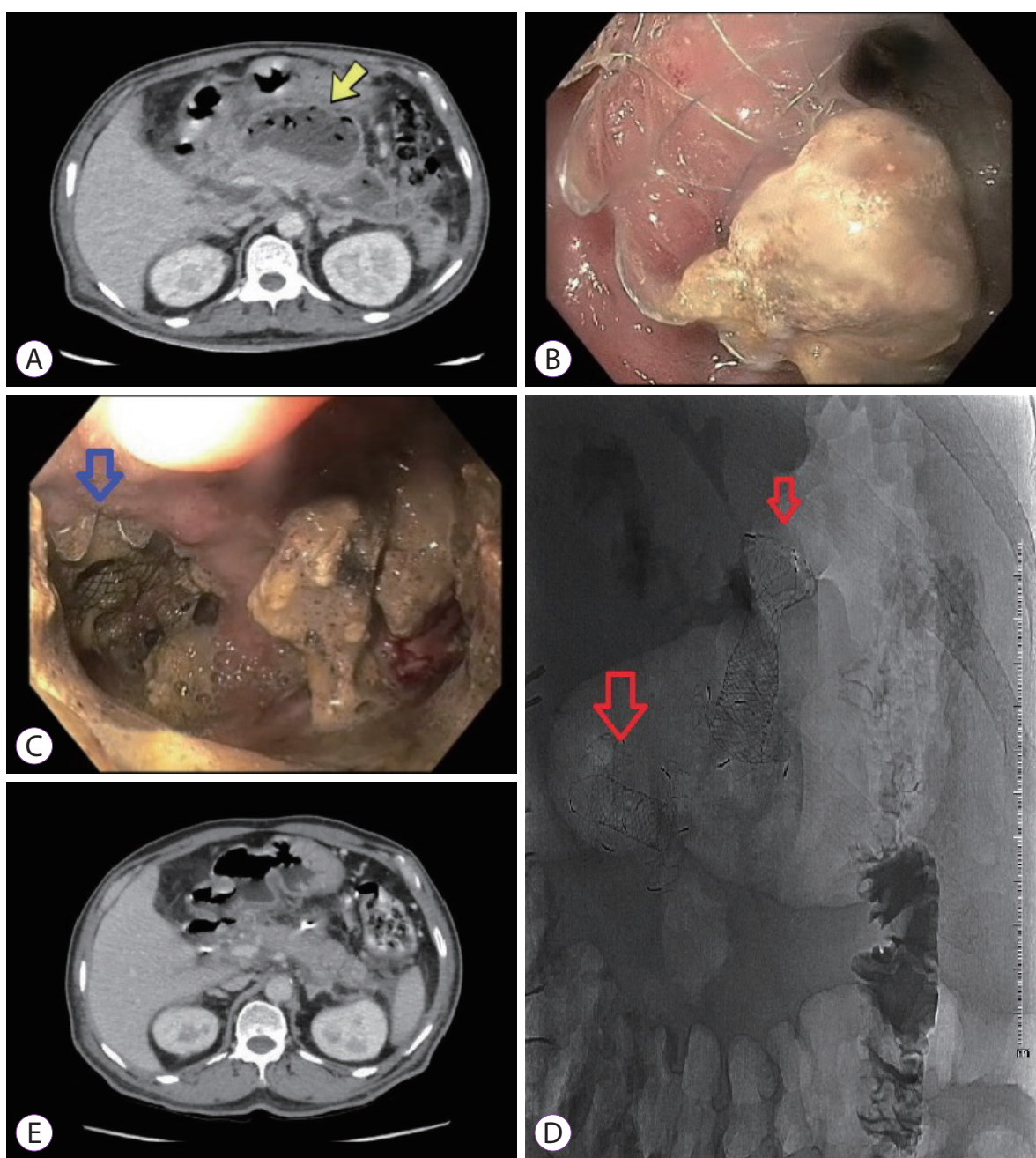

Fig. 2. (A) A computed tomography (CT) scan of the upper abdomen demonstrated an infected walled-off necrosis (WON) by showing air bubbles inside the cavity (yellow arrow). (B) Necrotic debris occluding the first lumen-apposing stent that was placed transduodenally. (C) Endoscopic debridement via the second lumen-apposing metallic stent (LAMS) that was placed transgastrically. Notably, the first LAMS (blue arrow) could be seen from a far distance. (D) Plain radiography of the abdomen demonstrated two LAMS (red arrows) in place at the WON site. (E) The final CT scan of the upper abdomen showed a resolution of the WON with a transmural plastic stent in place. 
group from South Korea demonstrated that after the removal of the drainage catheter with either external or internal stents, patients with main pancreatic duct disruptions had more recurrences of fluid collection than those without duct disruption (71\% vs. $17 \%)$ and that they were more likely to require surgery ( $43 \%$ vs. $6 \%){ }^{16}$ Therefore, the status of the pancreatic duct needs to be confirmed before the removal of a transmural stent.

Clinical evidence before completing EN are arbitrary concepts. Some experts recommended using computed tomography (CT) scans of the abdomen to demonstrate almost complete resolution of fluid collection in conjunction with clinical response, ${ }^{17}$ while others suggested more than $40 \%$ of necrotic tissue disappearance in 1 month as an endpoint. ${ }^{18}$ The Mayo Clinic team has proposed the endpoint of EN as the demonstration of granulation pink tissue in almost all walls of the cavity, which is usually achieved after complete debridement, and confirmation via CT scan of significant cavity reduction. ${ }^{19}$ Our group accepted the idea of seeing pink granulation tissue on the cavity wall in combination with clinical response, as this is the best way to demonstrate almost complete removal of the necrotic tissue. ${ }^{3}$

\section{ADVERSE EVENTS}

Regarding adverse events related to endoscopic treatment, complications were usually stent-related adverse events such as stent occlusion and stent-induced bleeding and migration, which are the most common. Wang et al. reported on the adverse events in three different types of stents: PSs, LAMSs, and fully covered self-expandable metallic stents. ${ }^{20}$ They found no difference in the proportion of stent-related adverse events between stent types except LAMSs, which had the lowest risk of stent migration. ${ }^{19}$ Stent occlusion and migration are manageable with endoscopy and rarely require surgery. In contrast, bleeding from the development of a pseudoaneurysm is indicated for angiographic embolization, which was the main cause of mortality in that series. ${ }^{20}$

Another report showed a low rate of self-limited bleeding in 5 out of 68 patients (7.3\%) with WON after LAMS placement. ${ }^{21}$ A recent meta-analysis with heterogeneous patients pooled from 11 studies ( $n=688$ ) also confirmed the superiority of a LAMS over a PS in terms of lower rates of adverse events and re-intervention. ${ }^{22}$

\section{CONCLUSIONS}

Before considering an endoscopic approach for patients with WON, the patient's age and the location and symptoms related to WON are the main parameters to be considered before offering treatment. Currently, a step-up strategy is recommended, particularly when a PS is selected as the drainage device. Over many years, there has been an evolution in stent selection, from PSs to metallic stents. LAMSs are gaining more popularity as they can offer direct EN in fewer sessions than PSs. In addition, the MTGT may be performed in a stepup strategy if only one stent fails to achieve debris clearance.

\section{Conflicts of Interest}

The author has no financial conflicts of interest.

\section{Acknowledgement}

This research received funding from a grant for International Research Integration: Chula Research Scholar; Ratchadaphiseksomphot Endowment Fund, Chulalongkorn University, and was supported by the Center of Excellence for Innovation and Endoscopy in Gastrointestinal Oncology, Faculty of Medicine, Chulalongkorn University.

\section{REFERENCES}

1. Mouli VP, Sreenivas V, Garg PK. Efficacy of conservative treatment, without necrosectomy, for infected pancreatic necrosis: a systematic review and meta-analysis. Gastroenterology 2013;144:333-340.e2.

2. Rana SS, Bhasin DK, Nanda M, et al. Endoscopic transpapillary drainage for external fistulas developing after surgical or radiological pancreatic interventions. J Gastroenterol Hepatol 2010;25:1087-1092.

3. Isayama $\mathrm{H}$, Nakai $\mathrm{Y}$, Rerknimitr R, et al. Asian consensus statements on endoscopic management of walled-off necrosis. Part 2: endoscopic management. J Gastroenterol Hepatol 2016;31:1555-1565.

4. Banks PA, Bollen TL, Dervenis C, et al. Classification of acute pancreatitis--2012: revision of the Atlanta classification and definitions by international consensus. Gut 2013;62:102-111.

5. Warshaw AL, Rattner DW. Timing of surgical drainage for pancreatic pseudocyst. Clinical and chemical criteria. Ann Surg 1985;202:720-724.

6. Lakhtakia S, Basha J, Talukdar R, et al. Endoscopic "step-up approach" using a dedicated biflanged metal stent reduces the need for direct necrosectomy in walled-off necrosis (with videos). Gastrointest Endosc 2017;85:1243-1252.

7. Rana SS, Sharma V, Sharma R, Gupta R, Bhasin DK. Endoscopic ultrasound guided transmural drainage of walled off pancreatic necrosis using a "step - up" approach: a single centre experience. Pancreatology 2017;17:203-208.

8. Nemoto Y, Attam R, Arain MA, et al. Interventions for walled off necrosis using an algorithm based endoscopic step-up approach: outcomes in a large cohort of patients. Pancreatology 2017;17:663-668.

9. Park DH, Lee SS, Moon SH, et al. Endoscopic ultrasound-guided versus conventional transmural drainage for pancreatic pseudocysts: a prospective randomized trial. Endoscopy 2009;41:842-848.

10. Fagenholz PJ, Thabet A, Mueller PR, Forcione DG. Combined endoscopic trangastric drainage and video assisted retroperitoneal pancreatic debridement - the best of both worlds for extensive pancreatic necrosis with enteric fistulae. Pancreatology 2016;16:788-790.

11. Bakker OJ, van Santvoort HC, van Brunschot S, et al. Endoscopic transgastric vs surgical necrosectomy for infected necrotizing pancreatitis: a randomized trial. JAMA 2012;307:1053-1061.

12. Varadarajulu S, Phadnis MA, Christein JD, Wilcox CM. Multiple transluminal gateway technique for EUS-guided drainage of symptom- 
atic walled-off pancreatic necrosis. Gastrointest Endosc 2011;74:74-80.

13. Chen YI, Yang J, Friedland S, et al. Lumen apposing metal stents are superior to plastic stents in pancreatic walled-off necrosis: a large international multicenter study. Endosc Int Open 2019;7:E347-E354.

14. Mohan BP, Jayaraj M, Asokkumar R, et al. Lumen apposing metal stents in drainage of pancreatic walled-off necrosis, are they any better than plastic stents? A systematic review and meta-analysis of studies published since the revised Atlanta classification of pancreatic fluid collections. Endosc Ultrasound 2019;8:82-90.

15. Yan L, Dargan A, Nieto J, et al. Direct endoscopic necrosectomy at the time of transmural stent placement results in earlier resolution of complex walled-off pancreatic necrosis: results from a large multicenter United States trial. Endosc Ultrasound 2019;8:172-179.

16. Jang JW, Kim MH, Oh D, et al. Factors and outcomes associated with pancreatic duct disruption in patients with acute necrotizing pancreatitis. Pancreatology 2016;16:958-965.

17. Papachristou GI, Takahashi N, Chahal P, Sarr MG, Baron TH. Peroral endoscopic drainage/debridement of walled-off pancreatic necrosis. Ann Surg 2007;245:943-951.
18. Gornals JB, De la Serna-Higuera C, Sánchez-Yague A, Loras C, Sánchez-Cantos AM, Pérez-Miranda M. Endosonography-guided drainage of pancreatic fluid collections with a novel lumen-apposing stent. Surg Endosc 2013;27:1428-1434.

19. Gardner TB, Chahal P, Papachristou GI, et al. A comparison of direct endoscopic necrosectomy with transmural endoscopic drainage for the treatment of walled-off pancreatic necrosis. Gastrointest Endosc 2009;69:1085-1094.

20. Wang Z, Zhao S, Meng Q, et al. Comparison of three different stents for endoscopic ultrasound-guided drainage of pancreatic fluid collection: a large retrospective study. J Gastroenterol Hepatol 2019;34:791-798.

21. Siddiqui AA, Adler DG, Nieto J, et al. EUS-guided drainage of peripancreatic fluid collections and necrosis by using a novel lumen-apposing stent: a large retrospective, multicenter U.S. experience (with videos). Gastrointest Endosc 2016;83:699-707.

22. Hammad T, Khan MA, Alastal Y, et al. Efficacy and safety of lumen-apposing metal stents in management of pancreatic fluid collections: are they better than plastic stents? A systematic review and meta-analysis. Dig Dis Sci 2018;63:289-301. 